Research Article

\title{
CTRP3 Protects against High Glucose-Induced Cell Injury in Human Umbilical Vein Endothelial Cells
}

\author{
Fang Wang $\mathbb{D}^{1},{ }^{1}$ Linlin Zhao, ${ }^{1}$ Yingguang Shan, ${ }^{2}$ Ran $\mathrm{Li}_{\mathbb{D}},{ }^{2}$ and Guijun Qin $\mathbb{D}^{1}$ \\ ${ }^{1}$ Department of Endocrinology, The First Affiliated Hospital of Zhengzhou University, Zhengzhou, China \\ ${ }^{2}$ Department of Cardiology, The First Affiliated Hospital of Zhengzhou University, Zhengzhou, China
}

Correspondence should be addressed to Ran Li; fcclir2@zzu.edu.cn and Guijun Qin; hyqingj@zzu.edu.cn

Received 19 March 2019; Accepted 12 June 2019; Published 24 July 2019

Academic Editor: Silvia Cantara

Copyright (c) 2019 Fang Wang et al. This is an open access article distributed under the Creative Commons Attribution License, which permits unrestricted use, distribution, and reproduction in any medium, provided the original work is properly cited.

\begin{abstract}
Aims. Inflammation was closely associated with diabetes-related endothelial dysfunction. C1q/tumor necrosis factor-related protein 3 (CTRP3) is a member of the CTRP family and can provide cardioprotection in many cardiovascular diseases via suppressing the production of inflammatory factors. However, the role of CTRP3 in high glucose- (HG-) related endothelial dysfunction remains unclear. This study evaluates the effects of CTRP3 on HG-induced cell inflammation and apoptosis. Materials and Methods. To prevent high glucose-induced cell injury, human umbilical vein endothelial cells (HUVECs) were pretreated with recombinant CTRP3 for 1 hour followed by normal glucose $(5.5 \mathrm{mmol} / \mathrm{l})$ or high glucose $(33 \mathrm{mmol} / \mathrm{l})$ treatment. After that, cell apoptosis and inflammatory factors were determined. Results. Our results demonstrated that CTRP3 mRNA and protein expression were significantly decreased after HG exposure in HUVECs. Recombinant human CTRP3 inhibited HG-induced accumulation of inflammatory factors and cell loss in HUVECs. CTRP3 treatment also increased the phosphorylation levels of protein kinase $\mathrm{B}(\mathrm{AKT} / \mathrm{PKB})$ and the mammalian target of rapamycin (mTOR) in HUVECs. CTRP3 lost its inhibitory effects on HG-induced cell inflammation and apoptosis after AKT inhibition. Knockdown of endogenous CTRP3 in HUVECs resulted in increased inflammation and decreased cell viability in vitro. Conclusions. Taken together, these findings indicated that CTRP3 treatment blocked the accumulation of inflammatory factors and cell loss in HUVECs after HG exposure through the activation of AKT-mTOR signaling pathway. Thus, CTRP3 may be a potential therapeutic drug for the prevention of diabetes-related endothelial dysfunction.
\end{abstract}

\section{Introduction}

Hyperglycemia is one of the major causes of vascular complications in patients with diabetes $[1,2]$. Diabetes-related endothelial dysfunction contributed to the development of diabetic vascular complications [3]. Endothelial dysfunction could be observed in the initial stage of diabetes [4]. Sustained high glucose $(\mathrm{HG})$ induced profound production of inflammatory factors in endothelial cells, thus resulting in the death of the endothelium and apoptosis $[5,6]$. Shortterm high glucose exposure increased monocyte chemotactic protein 1 (MCP-1) expression in human aortic endothelial cells to enhance monocyte-endothelial cell adhesion [7]. Therefore, to prevent diabetes-related endothelial dysfunction, it is imperative to find molecules that could inhibit HG-induced inflammation and cell apoptosis.
C1q/tumor necrosis factor-related proteins (CTRPs) are a highly conserved family of adiponectin paralogs. CTRP3, also called CORS-26, has been reported to be highly expressed in cardiac samples [8,9]. CTRP3 is composed of four domains, including an N-terminal domain, a short variable domain, a collagen-like domain, and a C-terminal globular domain [10]. Globular CTRP3 (lacking parts at the $\mathrm{N}$-terminal domain) was used frequently to investigate the biological functions of CTRP3 $[9,11]$. Ma et al. found that CTRP3 improved cardiac dysfunction and prevented diabetes-related cardiac injury in rats [9]. Previous studies have found that CTRP3 suppressed cardiomyocyte apoptosis and prevented cardiac fibrosis in ischemic mouse hearts [12]. CTRP3 also attenuated doxorubicin-induced cardiac death and reduced sepsis-induced myocardial dysfunction in mice $[11,13]$. Notably, CTRP3 could act as a novel endogenous 
antagonist of LPS and prevent LPS-related inflammation [14]. However, there is still a paucity of data regarding the effects of CTRP3 on HG-induced endothelial dysfunction.

Therefore, we conducted the present study to explore the potential effects of CTRP3 on diabetes-related endothelial dysfunction in human umbilical vein endothelial cells (HUVECs). We found that CTRP3 was downregulated in HUVECs after high glucose treatment and that CTRP3 administration reduced high glucose-induced cell inflammation and cell death via activating the protein kinase $B$ (AKT/PKB) signaling pathway.

\section{Methods and Materials}

2.1. Reagents. Recombinant CTRP3 globular form (human) was obtained from Aviscera Bioscience (CA, USA). The following primary antibodies were purchased from Abcam (Boston, MA, USA): anti-CTRP3 antibody $(1: 1000$, ab36870), anti-glyceraldehyde 3-phosphate dehydrogenase (GAPDH, 1:1000, ab181602), anti-nuclear factor- $\kappa \mathrm{B}$ (NF- $\kappa$ B, phospho S536) antibody (1:1000, ab86299), anti-NF- $\kappa$ B p65 antibody $(1: 1000$, ab16502), antiproliferating cell nuclear antigen (PCNA, 1:1000, ab92552), anti-Bax (ab32503, $1: 1000)$, anti-B-cell lymphoma 2 (Bcl-2, ab196495, 1:1000), anti-AKT (phospho T308, 1:1000, ab38449), anti-AKT (1:1000, ab8805), anti-mammalian target of rapamycin (p-mTOR, 1:1000, ab137133, phospho S2481), and anti-mTOR $(1: 1000$, ab2732). A specific AKT1/2 kinase inhibitor was obtained from Sigma-Aldrich (No. A6730, St. Louis, MO, USA). Isolation of nuclear proteins was performed using a kit from Beyotime Biotechnology (No. P0028, Beijing, China).

2.2. Cell Culture and Treatment. HUVECs were obtained from the American Type Culture Collection (Manassas, VA, USA). This cell line was grown in Dulbecco's modified Eagle's medium (DMEM) supplemented with $10 \%$ fetal bovine serum at $37^{\circ} \mathrm{C}$ in a $5 \% \mathrm{CO}_{2}$ humidified atmosphere. To induce high glucose-induced cell injury, cells were cultured in DMEM supplemented with $30 \mathrm{mmol} / \mathrm{l}$ glucose. The cells in the control group were cultured in the presence of $5 \mathrm{mmol} / \mathrm{l}$ glucose. To investigate the protective role of CTRP3, HUVECs were pretreated with recombinant CTRP3 $(3 \mu \mathrm{g} / \mathrm{ml})$ for 1 hour followed by normal glucose $(5.5 \mathrm{mmol} / \mathrm{l})$ or high glucose $(33 \mathrm{mmol} / \mathrm{l})$ treatment. The dose of CTRP3 was determined according to a previous study [9]. To confirm the requirement of AKT activation in CTRP3-provided protection, HUVECs were incubated with AKT1/2 kinase inhibitor $(1 \mu \mathrm{mol} / \mathrm{l})$. To determine the role of endogenous CTRP3, cells were incubated with siCTRP3 $(50 \mathrm{mmol} / \mathrm{l})$ for $24 \mathrm{~h}$ to knock down the expression of CTRP3 and siRNA was used as a negative control.

2.3. Western Blot. HUVECs were lysed in RIPA buffer containing phenylmethylsulfonyl fluoride (PMSF, $1 \mathrm{mmol} / \mathrm{l}$ ) and phosphatase inhibitors $(0.01 \mathrm{mmol} / \mathrm{l})$. The protein concentration was determined by a bicinchoninic acid (BCA) protein assay (Beyotime Biotechnology, China). For each detection, proteins $(20 \mu \mathrm{g})$ were separated by $6 \%$ (mTOR) or 10\% SDS-PAGE and electrotransferred onto PVDF membranes (Millipore, Billerica, MA, USA). After blocking with $5 \%$ nonfat milk, the membranes were reacted with primary antibodies $\left(4^{\circ} \mathrm{C}\right.$ overnight $)$ and then reacted with the secondary antibody (room temperature for $1 \mathrm{~h}$ ). After that, the membranes were examined using the enhanced chemiluminescence reagents (Amersham, Buckinghamshire, UK). The intensity of the band was counted using the ImageJ software.

2.4. Quantitative Real-Time Polymerase Chain Reaction Assay. Total RNA from the HUEVCs was extracted using TRIzol reagent (Invitrogen, United States) [15]. Then, the purity and concentration of the RNA were detected. After that, total RNA was reverse-transcribed to cDNA using the PrimeScript ${ }^{\mathrm{TM}}$ RT Reagent Kit with gDNA Eraser. The quantification of real-time PCR was performed using an ABI Prism 7700 Real-Time PCR System (Applied Biosystems, Foster City, CA) with the SYBR Green PCR Kit (Takara). GAPDH was used as the reference control.

2.5. TUNEL Staining. To detect cell apoptosis, HUVECs were seeded into a 24-well plate at an intensity of $5 \times 10^{4} \mathrm{cells} / \mathrm{cm}^{2}$. After HG exposure for 72 hours, TUNEL assay was also conducted using a transferase-mediated dUTP nick-end labeling (TUNEL) kit (Roche, Germany) according to the manufacturer's instructions.

2.6. Cell Viability Assay. HUVECs were seeded into 96-well plates at an intensity of $5 \times 10^{4} \mathrm{cells} / \mathrm{cm}^{2}$. After HG exposure for 72 hours, cell viability was measured using a Cell Counting Kit-8 (CCK-8, Dojindo Laboratories, Kumamoto, Japan). The optical density (OD) values were evaluated at the wavelength of $550 \mathrm{~nm}$ using a microplate reader.

2.7. Statistical Analysis. All the data were expressed as the mean \pm standard deviation (SD). Statistical analysis among multiple groups was carried out using one-way analysis of variance followed by Tukey post hoc. We used the Student $t$-test to compare significance between two groups.

\section{Result}

3.1. CTRP3 Was Downregulated by High Glucose in HUVECs. To evaluate the role of CTRP3 in high glucose-induced endothelial dysfunction, we first detected CTRP3 expression in HUVECs. Using quantitative real-time PCR assay, we found that CTRP3 mRNA gradually decreased at different time points $(6,12$, and $24 \mathrm{~h})$ at a glucose concentration of $33 \mathrm{mmol} / \mathrm{l}$ in HUVECs (Figure 1(a)). Similarly, western blot analysis showed that CTRP3 protein expression was also decreased by high glucose exposure for $24 \mathrm{~h}$ in HUVECs (Figure 1(b)).

3.2. CTRP3 Treatment Inhibited the Accumulation of Inflammatory Factors in HUVECs after HG Exposure. To examine the effect of CTRP3 on the inflammation induced by HG, HUVECs were subjected to recombinant human CTRP3 globular form for $24 \mathrm{~h}$. HG stimulation increased the expression of inflammatory factors, such as tumor necrosis factor- $\alpha$ (TNF- $\alpha$ ), interleukin-1 $\beta$ (IL- $1 \beta$ ), and MCP-1. 


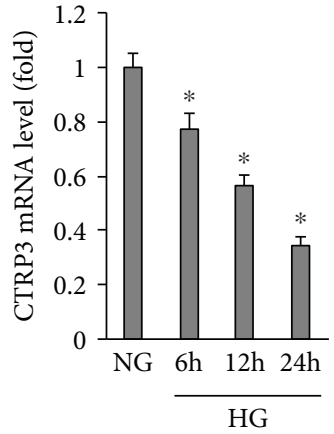

(a)

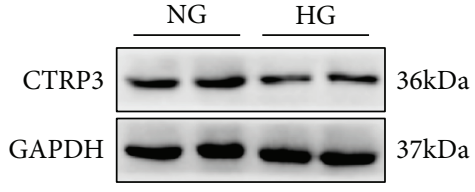

(b)

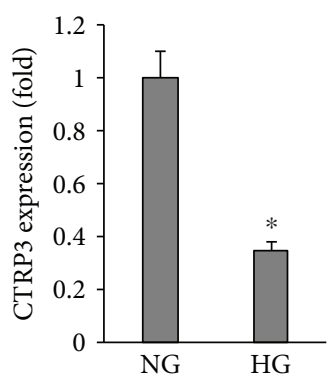

(c)

FIGURE 1: CTRP3 expression in HUVECs exposed to high glucose. (a) CTRP3 mRNA levels in HUVECs exposed to HG for indicated different times $(n=5)$. (b) Protein levels of CTRP3 expressed in HUVECs exposed to HG for 24 hours $(n=5)$. Data are expressed as mean \pm SD of five independent experiments. ${ }^{*} P<0.05$ when compared to the NG group.

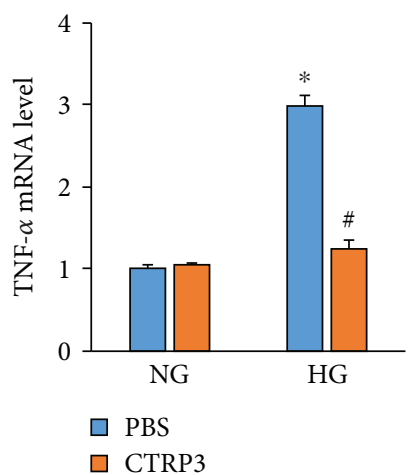

(a)

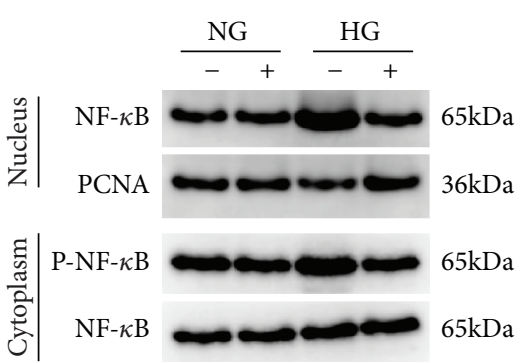

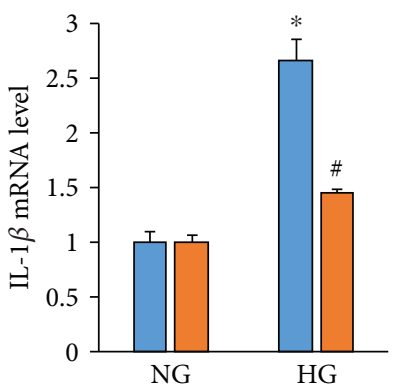

$\square$ PBS

$\square$ CTRP3

(b)

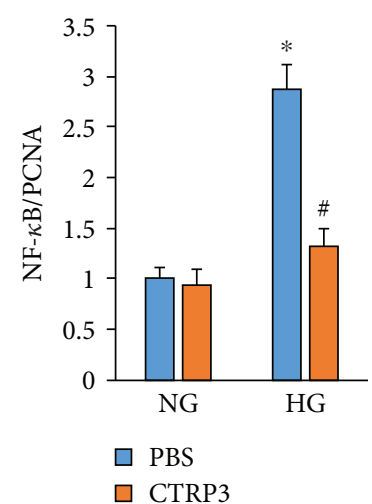

(d)

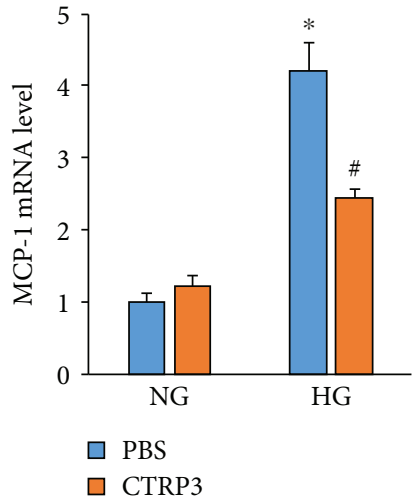

(c)

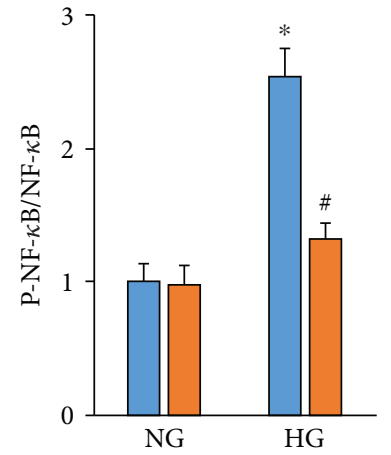
FIGURE 2: CTRP3 inhibits cell inflammation level in HUVECs induced by high glucose. $(\mathrm{a}-\mathrm{c})$ The mRNA expression of inflammatory factors
in HUVECs exposed to high glucose $(n=5)$. (d) The protein expression of NF- $\kappa$ B was detected by western blot analysis $(n=5)$. Data are expressed as mean $\pm \mathrm{SD}$ of five independent experiments. ${ }^{*} P<0.05$ when compared to the NG group; ${ }^{\#} P<0.05$ when compared to the HG group.

However, these pathological elevations were suppressed by the treatment of CTRP3 (Figures 2(a)-2(c)). Nuclear $\mathrm{NF}-\kappa \mathrm{B}$ levels in cells with or without CTRP3 were determined by western blot. High glucose-induced nuclear translocation of NF- $\kappa$ B was inhibited by CTRP3 (Figure 2(d)). High glucose-triggered upregulation of $\mathrm{p}-\mathrm{NF}-\kappa \mathrm{B}$ in the cytoplasm was lower in cells treated with $\mathrm{HG}+\mathrm{CTRP} 3$ compared with cells treated with HG alone (Figure 2(d)). 


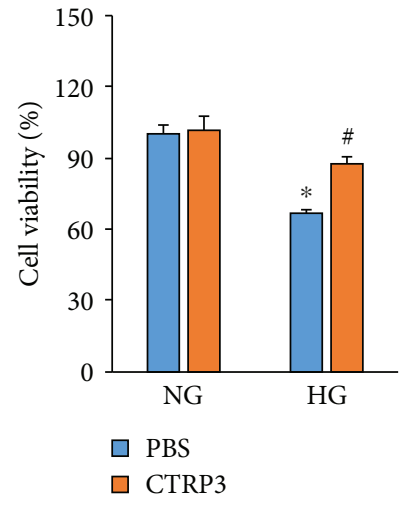

(a)

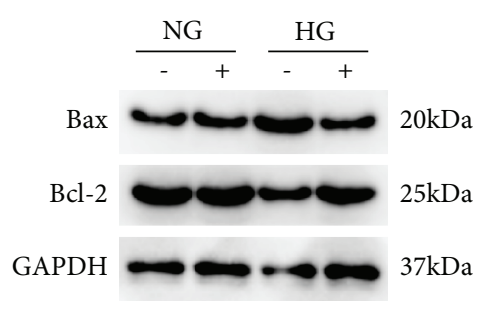

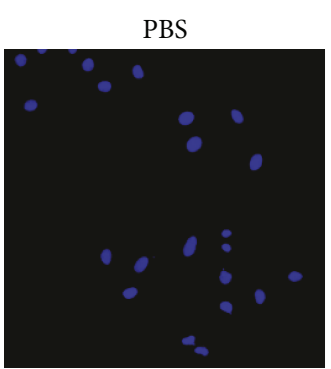
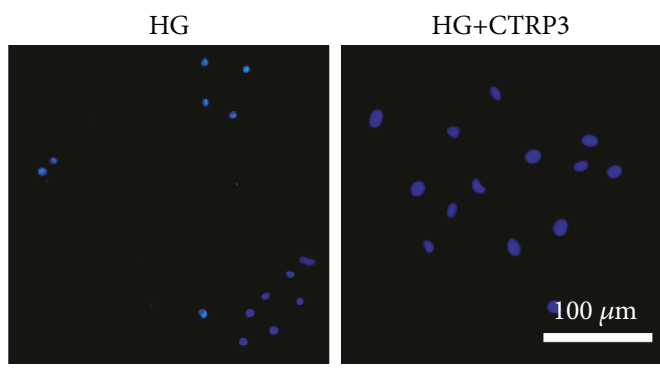

(b)
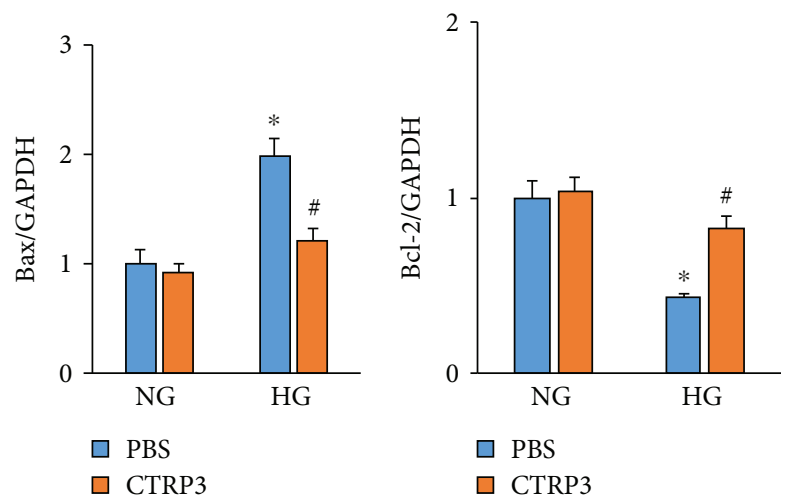

(c)

FIGURE 3: CTRP3 reduces the level of apoptosis in HUVECs induced by high glucose. (a) Cell viability in HUVECs induced by high glucose $(n=5)$. (b) Cell apoptosis as detected by TUNEL staining. (c) The protein expression of Bax and Bcl-2 was detected by western blot analysis $(n=5)$. Data are expressed as mean $\pm \mathrm{SD}$ of five independent experiments. ${ }^{*} P<0.05$ when compared to the NG group; ${ }^{\#} P<0.05$ when compared to the HG group.

3.3. CTRP3 Provided Antiapoptosis Effect in HUVECs after HG Exposure. The data in our study indicated that compared with the NG group, HG treatment markedly decreased cell viability of HUVECs. However, CTRP3 treatment almost restored cell viability to the normal level (Figure 3(a)). As shown in Figure 3(b), 72 hours post HG exposure, an increased level of apoptosis was observed in HG-treated cells and CTRP 3 administration could decrease the proportion of TUNEL-positive cells (Figure 3(b)). Western blot results showed that CTRP3 treatment upregulated $\mathrm{Bcl}-2$ protein expression and downregulated Bax protein expression in HUVECs after HG exposure (Figure 3(c)). However, there was no change in these apoptosis-related proteins between the NG+PBS group and the NG+CTRP3 group (Figure 3(c)).

3.4. CTRP3 Could Activate AKT-mTOR Signaling Pathway in HUVECs after HG Exposure. To explore the mechanism by which CTRP3 inhibited inflammation and cell apoptosis, we first detected the alteration in AKT-mTOR signaling pathway in HUVECs after HG exposure. There was no difference in the phosphorylation levels of AKT and mTOR between the NG+PBS and NG+CTRP3 groups (Figures 4(a)-4(c)). HG exposure markedly decreased the phosphorylation levels of AKT-mTOR in HUVECs; however, CTRP3 treatment restores the phosphorylation levels of AKT-mTOR (Figures 4(a)-4(c)). Next, we examined whether the activation of AKT-mTOR signaling pathway was involved in CTRP3-provided protection. The data in our study demonstrated that the AKT inhibitor was capable of blocking the protective effects of CTRP3 on cell viability and inflammation in HUVECs after HG exposure (Figures 4(d) and 4(e)).

3.5. CTRP3 Deletion Increased the Level of TNF- $\alpha$ and Decreased Cell Viability at Baseline. Next, we investigated whether the endogenous CTRP3 deficiency affected HUVECs. We knocked down CTRP3 in HUVECs. Downregulation of CTRP3, confirmed by western blot analysis (Figure 5(a)), decreased the phosphorylation levels of AKT in HUVECs (Figure 5(b)). CTRP3 deficiency increased the mRNA level of TNF- $\alpha$ and decreased the viability of HUVECs in basal conditions (Figures 5(c) and 5(d)).

\section{Discussion}

In this study, our results demonstrated that CTRP3 mRNA and protein expression were significantly decreased in HG-treated HUVECs. Using recombinant human CTRP3 globular form, we found that CTRP3 administration inhibited HG exposure-induced inflammatory factor accumulation 


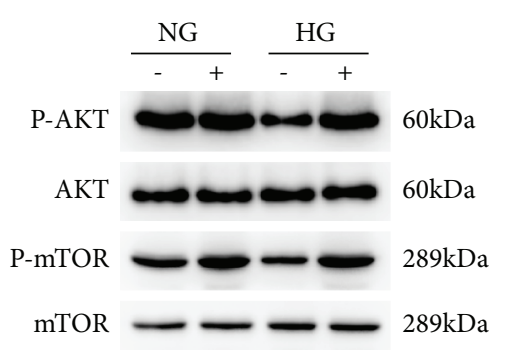

(a)

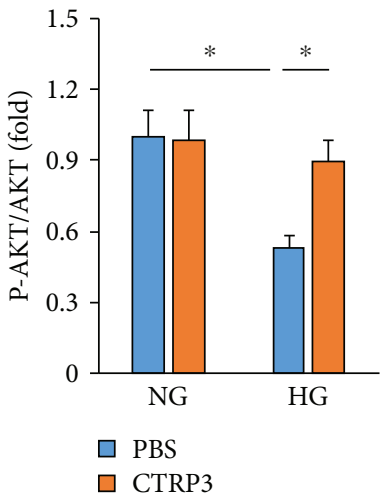

(b)

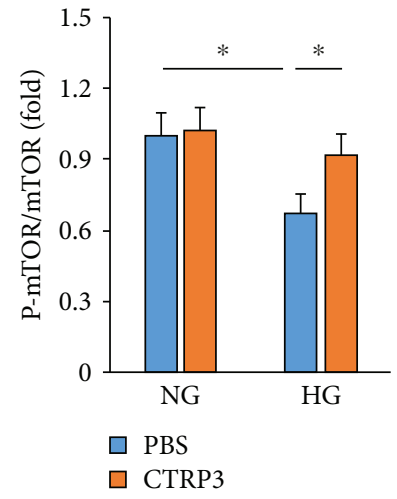

(c)

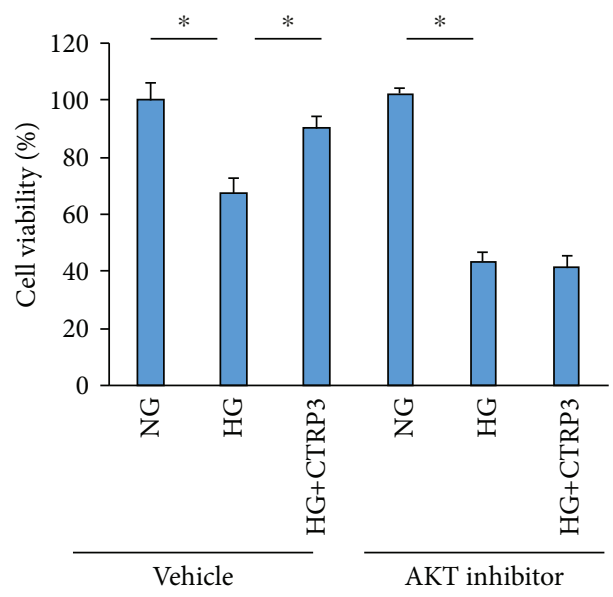

(d)

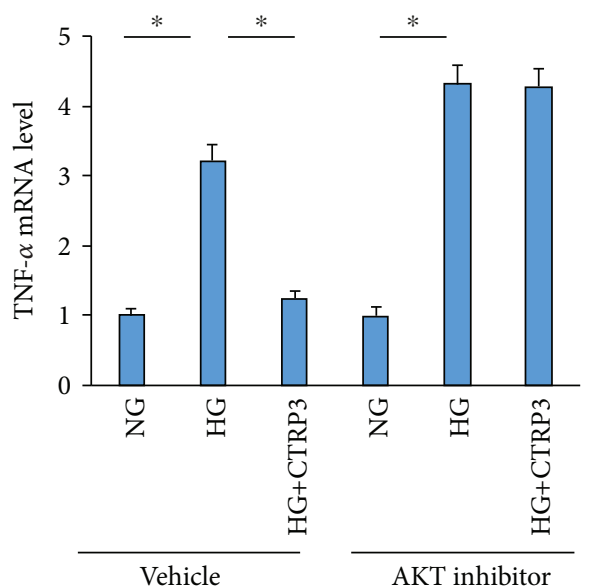

(e)

FIgURE 4: CTRP3 activated the AKT signaling pathway. (a-c) The protein expression of AKT and mTOR was detected by western blot analysis $(n=5)$. (d) Cell viability in HUVECs induced by high glucose $(n=5)$. (e) The mRNA expression of TNF- $\alpha$ in HUVECs exposed to high glucose $(n=5)$. Data are expressed as mean \pm SD of five independent experiments. ${ }^{*} P<0.05$ when compared to the matched control.

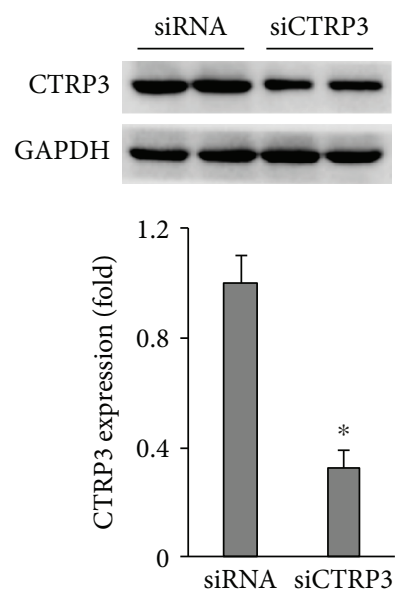

(a)

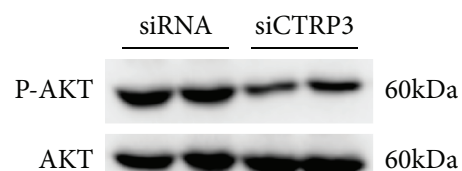

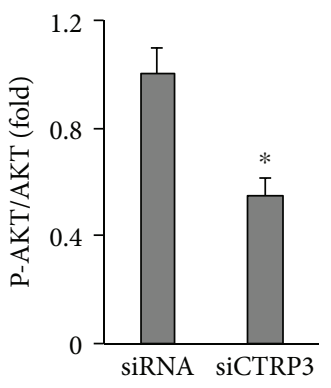

(b)

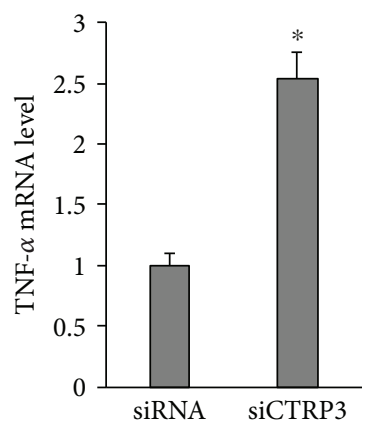

(c)

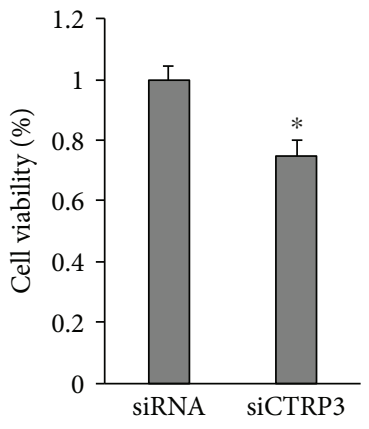

(d)

FIGURE 5: CTRP3 deficiency increased inflammation and cell death at baseline. (a) Protein level of CTRP3 ( $n=5$ ). (b) The protein expression of AKT was detected by western blot analysis $(n=5)$. (c) The mRNA expression of inflammatory factor in HUVECs $(n=5)$. (d) Cell viability in HUVECs $(n=5)$. Data are expressed as mean \pm SD of five independent experiments. ${ }^{*} P<0.05$ when compared to the matched control. 
and cell loss in HUVECs. Mechanistically, CTRP3 could activate the AKT-mTOR signaling pathway in HUVECs, and AKT inhibition could abolish the protective effects provided by CTRP3 treatment. Knockdown of CTRP3 resulted in inflammation accumulation and cell death in vitro.

CTRP3 is an adipokine associated with various cardiovascular diseases, including diabetic cardiomyopathy, agentrelated cardiac injury, and cardiac hypertrophy $[9,11,16]$. The data in our study found that CTRP3 mRNA and protein expression were downregulated in the cells treated with HG, which was in line with the expression of CTRP3 in animals with myocardial ischemia and diabetes $[12,17]$. CTRP3 was also found to be decreased in obesity-related male reproductive dysfunction in mice [18]. However, there exists an opposite voice that CTRP3 mRNA and protein were increased in hypertrophic hearts [16]. The discrepancy between these studies might be explained by the different animal diseases and different pathological conditions.

The alteration of CTRP3 expression implies that CTRP3 might play a role in high glucose-induced endothelial dysfunction. As expected, CTRP3 treatment suppressed HG-induced inflammation accumulation and endothelial apoptosis in vitro. Our finding was in agreement with previous studies which described protective roles in cardiometabolic diseases $[11,12,18]$. However, Ma et al. found that CTRP3 overexpression promoted pressure overload which caused hypertrophic response in mice [16]. These incompatible results may be explained by the different intervention approaches.

The microinflammatory state is closely associated with the development of diabetes-related endothelial dysfunction [19]. High glucose could result in the excessive production of TNF- $\alpha$ and IL- $1 \beta$, which caused the activation of NF- $\kappa \mathrm{B}$ and led to amplification of inflammatory response [20]. Hyperglycemia also directly triggered the activation of $\mathrm{NF}-\kappa \mathrm{B}[21]$. After that, NF- $\kappa \mathrm{B}$ translocated into the nucleus and then regulated the expression of MCP-1 [22]. Forced expression of a dominant negative mutant of NF- $\kappa \mathrm{B}$ inhibited proinflammatory gene expression in endothelial cells [23]. Our study found that CTRP3 treatment reduced the mRNA levels of TNF- $\alpha$, IL- $1 \beta$, IL- 6 , and MCP- 1 in HUVECs after HG exposure. Sustained inflammation resulted in the apoptosis of the endothelium. Therefore, we detected the effect of CTRP3 on HG-induced endothelial apoptosis and found that CTRP3 also improved cell viability and reduced cell apoptosis in HG-treated HUVECs.

The AKT-mTOR signaling pathway was closely related with the development and progression of high glucoseinduced endothelial dysfunction [24, 25]. AKT, a serine/threonine protein kinase, is known as a focal point for signal transduction pathways responsible for cell survival [26]. AKT activation could promote cell viability by blocking Bax conformational change [27]. Therefore, we first detected the alteration of AKT in HG-treated HUVECs and found that phosphorylation levels of AKT and mTOR were significantly decreased after HG treatment. However, CTRP3 restored AKT phosphorylation to the normal level, which was consistent with a previous study [9]. In addition, we also found that CTRP3 reduced Bax expression and increased Bcl-2 expression in HUVECs. Ma et al. found that CTRP3 exerted its protection via AMP-activated protein kinase $\alpha$ $(\mathrm{AMPK} \alpha)$ not AKT signaling pathway [9]. To verify the hypothesis that CTRP3 provided protection against endothelial dysfunction via the activation of AKT signaling pathway, we used an AKT inhibitor. CTRP3 lost its protection against cell loss and inflammation after AKT inhibition, implying that CTRP3 attenuated HG-induced inflammation and apoptosis through the activation of the AKT-mTOR signaling pathway.

Collectively, the present study demonstrated that CTRP3 improves cell viability and ameliorates cell inflammation via activating the AKT pathway. Our study provides evidence for the application of CTRP3 in the treatment of high glucoseinduced endothelial dysfunction.

\section{Data Availability}

The data that support the findings of this study are available from the corresponding authors upon reasonable request.

\section{Conflicts of Interest}

No conflict of interests was stated by the authors.

\section{References}

[1] D. Dymkowska, B. Drabarek, P. Podszywalow-Bartnicka, J. Szczepanowska, and K. Zablocki, "Hyperglycaemia modifies energy metabolism and reactive oxygen species formation in endothelial cells in vitro," Archives of Biochemistry and Biophysics, vol. 542, pp. 7-13, 2014.

[2] S. M. Shenouda, M. E. Widlansky, K. Chen et al., "Altered mitochondrial dynamics contributes to endothelial dysfunction in diabetes mellitus," Circulation, vol. 124, no. 4, pp. 444-453, 2011.

[3] A. Taye, A. H. Saad, A. H. S. Kumar, and H. Morawietz, "Effect of apocynin on NADPH oxidase-mediated oxidative stressLOX-1-eNOS pathway in human endothelial cells exposed to high glucose," European Journal of Pharmacology, vol. 627, no. $1-3$, pp. $42-48,2010$.

[4] M. D. Siperstein, D. W. Foster, H. C. Knowles Jr., R. Levine, L. L. Madison, and J. Roth, "Control of blood glucose and diabetic vascular disease," The New England Journal of Medicine, vol. 296, no. 18, pp. 1060-1063, 1977.

[5] W. Fan, D. Han, Z. Sun et al., "Endothelial deletion of $\mathrm{mTORC1}$ protects against hindlimb ischemia in diabetic mice via activation of autophagy, attenuation of oxidative stress and alleviation of inflammation," Free Radical Biology \& Medicine, vol. 108, pp. 725-740, 2017.

[6] H. A. Toque, A. Fernandez-Flores, R. Mohamed, R. B. Caldwell, G. Ramesh, and R. W. Caldwell, "Netrin-1 is a novel regulator of vascular endothelial function in diabetes," PLoS One, vol. 12, no. 10, article e0186734, 2017.

[7] R. Piga, Y. Naito, S. Kokura, O. Handa, and T. Yoshikawa, "Short-term high glucose exposure induces monocyteendothelial cells adhesion and transmigration by increasing VCAM-1 and MCP-1 expression in human aortic endothelial cells," Atherosclerosis, vol. 193, no. 2, pp. 328-334, 2007. 
[8] J. Weigert, M. Neumeier, A. Schäffler et al., "The adiponectin paralog CORS-26 has anti-inflammatory properties and is produced by human monocytic cells," FEBS Letters, vol. 579, no. 25, pp. 5565-5570, 2005.

[9] Z. G. Ma, Y. P. Yuan, S. C. Xu et al., "CTRP3 attenuates cardiac dysfunction, inflammation, oxidative stress and cell death in diabetic cardiomyopathy in rats," Diabetologia, vol. 60, no. 6, pp. 1126-1137, 2017.

[10] U. Kishore, C. Gaboriaud, P. Waters et al., "C1q and tumor necrosis factor superfamily: modularity and versatility," Trends in Immunology, vol. 25, no. 10, pp. 551-561, 2004.

[11] Y. P. Yuan, Z. G. Ma, X. Zhang et al., "CTRP3 protected against doxorubicin-induced cardiac dysfunction, inflammation and cell death via activation of Sirt1," Journal of Molecular and Cellular Cardiology, vol. 114, pp. 38-47, 2018.

[12] W. Yi, Y. Sun, Y. Yuan et al., "C1q/tumor necrosis factorrelated protein-3, a newly identified adipokine, is a novel antiapoptotic, proangiogenic, and cardioprotective molecule in the ischemic mouse heart," Circulation, vol. 125, no. 25, pp. 3159-3169, 2012.

[13] W. Y. Wei, Z. G. Ma, N. Zhang et al., "Overexpression of CTRP3 protects against sepsis-induced myocardial dysfunction in mice," Molecular and Cellular Endocrinology, vol. 476, pp. 27-36, 2018.

[14] A. Kopp, M. Bala, C. Buechler et al., "C1q/TNF-related protein-3 represents a novel and endogenous lipopolysaccharide antagonist of the adipose tissue," Endocrinology, vol. 151, no. 11, pp. 5267-5278, 2010.

[15] Z. G. Ma, J. Dai, Y. P. Yuan et al., "T-bet deficiency attenuates cardiac remodelling in rats," Basic Research in Cardiology, vol. 113, no. 3, p. 19, 2018.

[16] Z. G. Ma, Y. P. Yuan, X. Zhang et al., "Clq-tumour necrosis factor-related protein-3 exacerbates cardiac hypertrophy in mice," Cardiovascular Research, vol. 115, no. 6, pp. 10671077, 2019.

[17] D. Wu, H. Lei, J. Y. Wang et al., "CTRP3 attenuates postinfarct cardiac fibrosis by targeting Smad3 activation and inhibiting myofibroblast differentiation," Journal of Molecular Medicine (Berlin, Germany), vol. 93, no. 12, pp. 1311-1325, 2015.

[18] Y. Mu, T. L. Yin, L. Yin, X. Hu, and J. Yang, "CTRP3 attenuates high-fat diet-induced male reproductive dysfunction in mice," Clinical Science (London, England), vol. 132, no. 8, pp. 883-899, 2018.

[19] Z. Li, Y. Li, J. M. Overstreet et al., "Inhibition of epidermal growth factor receptor activation is associated with improved diabetic nephropathy and insulin resistance in type 2 diabetes," Diabetes, vol. 67, no. 9, pp. 1847-1857, 2018.

[20] V. Rao, L. B. V. Rao, S. H. Tan, M. Candasamy, and S. K. Bhattamisra, "Diabetic nephropathy: an update on pathogenesis and drug development," Diabetes \& Metabolic Syndrome: Clinical Research \& Reviews, vol. 13, no. 1, pp. 754-762, 2019.

[21] D. Sharma, P. Bhattacharya, K. Kalia, and V. Tiwari, "Diabetic nephropathy: new insights into established therapeutic paradigms and novel molecular targets," Diabetes Research and Clinical Practice, vol. 128, pp. 91-108, 2017.

[22] G. H. Tesch, "MCP-1/CCL2: a new diagnostic marker and therapeutic target for progressive renal injury in diabetic nephropathy," American Journal of Physiology. Renal Physiology, vol. 294, no. 4, pp. F697-F701, 2008.
[23] M. P. Soares, A. Muniappan, E. Kaczmarek et al., "Adenovirusmediated expression of a dominant negative mutant of p65/RelA inhibits proinflammatory gene expression in endothelial cells without sensitizing to apoptosis," Journal of Immunology, vol. 161, no. 9, pp. 4572-4582, 1998.

[24] Z. Zhang, M. Jiang, X. Xie et al., "Oleanolic acid ameliorates high glucose-induced endothelial dysfunction via PPAR $\delta$ activation," Scientific Reports, vol. 7, no. 1, article 40237, 2017.

[25] H. Li, Y. Xu, R. Guan et al., "Icariside II prevents high-glucoseinduced injury on human cavernous endothelial cells through Akt-eNOS signaling pathway," Andrology, vol. 3, no. 2, pp. 408-416, 2015.

[26] J. Downward, "Mechanisms and consequences of activation of protein kinase B/Akt," Current Opinion in Cell Biology, vol. 10, no. 2, pp. 262-267, 1998.

[27] H. Yamaguchi and H. G. Wang, "The protein kinase PKB/Akt regulates cell survival and apoptosis by inhibiting Bax conformational change," Oncogene, vol. 20, no. 53, pp. 7779-7786, 2001. 


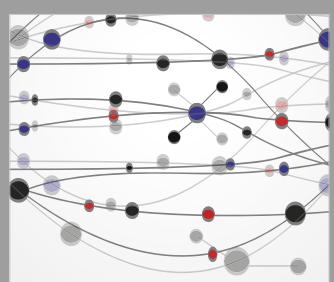

The Scientific World Journal
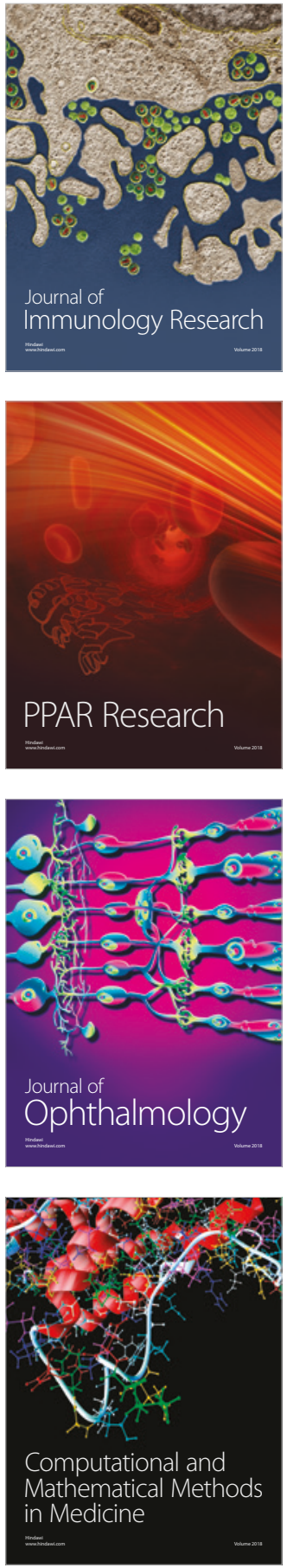

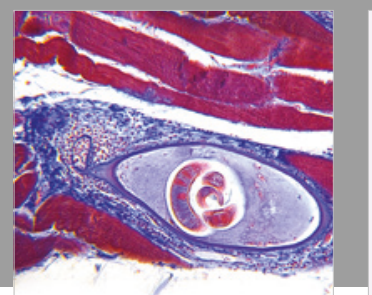

Gastroenterology Research and Practice

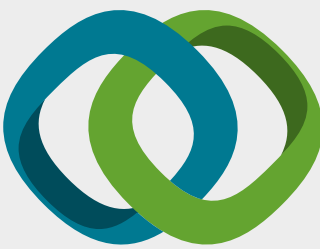

\section{Hindawi}

Submit your manuscripts at

www.hindawi.com
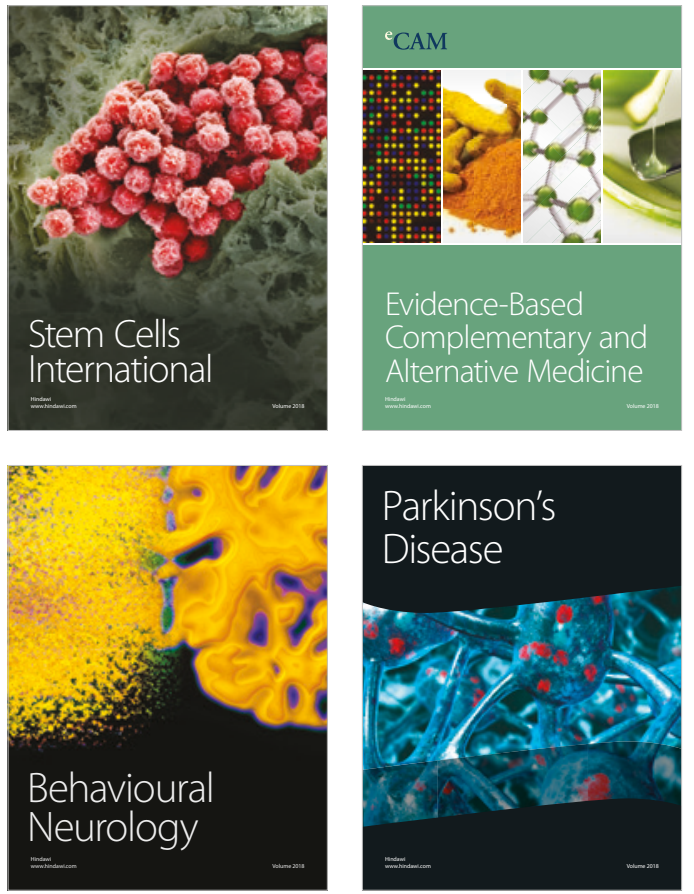

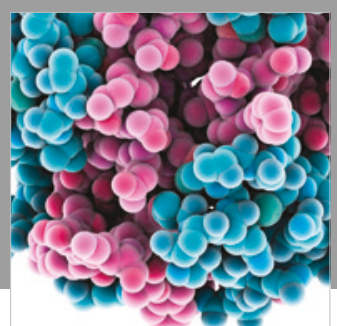

ournal of

Diabetes Research

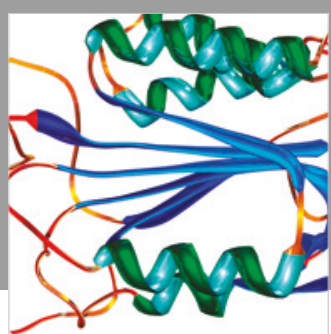

Disease Markers
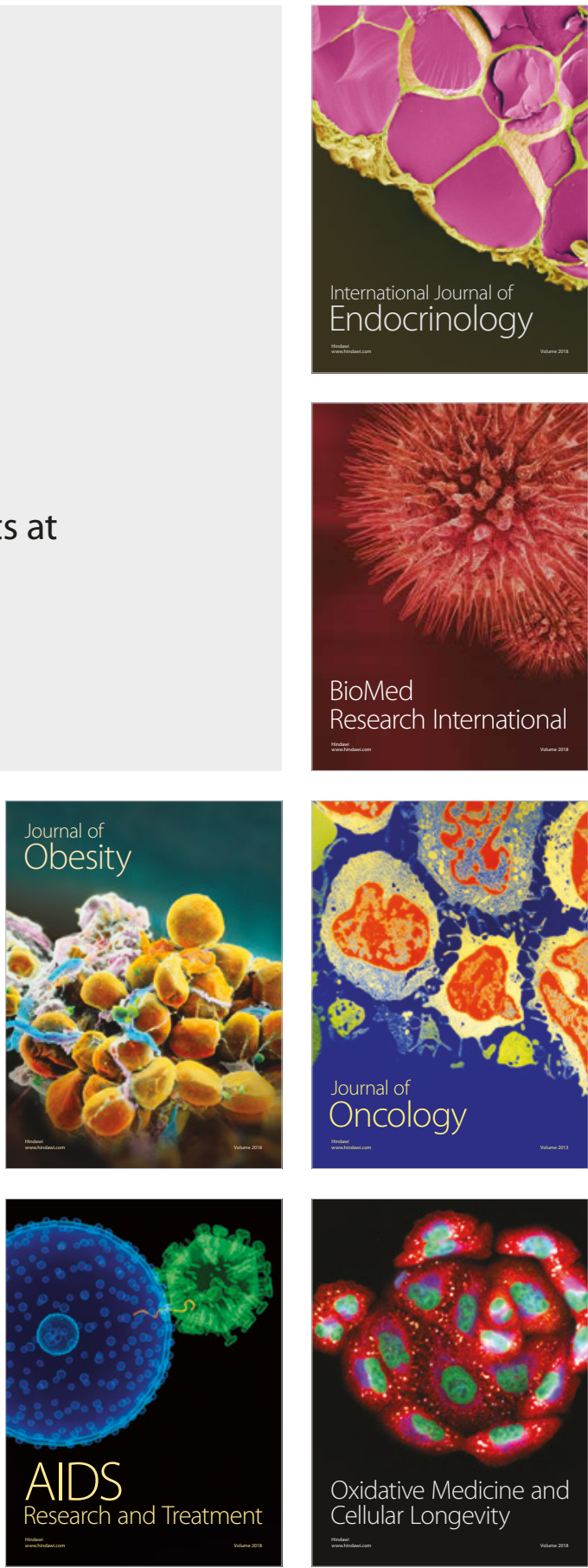